\title{
Frecuencia de coccidiosis y criptosporidiosis intestinal en pacien- tes diarreicos e infección con el virus de la inmunodeficiencia hu- mana, en un hospital de Chiclayo, Perú
}

\author{
Claudia Giuliana Larrea-Vargas ${ }^{1 a}$
}

\section{RESUMEN}

Objetivo: Determinar la frecuencia de coccidiosis y criptosporidiosis intestinal en pacientes diarreicos e infección con el virus de la inmunodeficiencia humana (VIH), admitidos en un hospital de Chiclayo, Perú; así como, relacionarlo con el tiempo de diagnóstico, cantidad linfocitos T CD4+ y carga viral. Material y Método. Estudio observacional, prospectivo y transversal analítico. La muestra fue de 34 pacientes con infección por VIH y diarrea, durante el periodo marzo a diciembre del 2015. Para la detección de parásitos se realizó examen directo, concentración por flotación Sheather Sugar y tinción Ziehl Neelsen modificado; y para los factores, análisis documental de registros. Resultados. 22 pacientes tuvieron parásitos intestinales; 11 con coccidios intestinales, Cystosospora belli (36 \%) y Cryptosporidium sp (14\%). No se observó asociación entre tiempo de diagnóstico $(p=0,157)$, cantidad linfocitos T CD4+ $(p=0,754)$ y carga viral $(p=1,000)$, y la coccidiosis y criptosporidiosis intestinal. Conclusiones. La coccidiosis y criptosporidiosis intestinal son infecciones frecuentes y que cuasan diarrea en pacientes con VIH en tiempos de Tratamiento Antirretroviral de gran actividad (TARGA).

Palabras clave: Coccidios, Cryptosporidium, Cystoisospora (Fuente: DeCS-BIREME)

\section{Frequency of intestinal coccidiosis and cryptosporidiosis in dia- rrheic patients and infection with the human immunodeficiency virus, to a hospital in Chiclayo, Peru}

\section{ABSTRACT}

Objective: To determine the frequency of intestinal coccidiosis and cryptosporidiosis in diarrheic patients and infection with the human immunodeficiency virus (HIV), admitted to a hospital in Chiclayo, Peru; as well as, relate it to the time of diagnosis, CD4 + T lymphocytes and viral load. Material and method. Observational, prospective and transversal analytical study. The sample consisted of 34 patients with HIV infection and diarrhea, during the period March to December 2015. For the detection of parasites, a direct examination was carried out, concentration by flotation Sheather Sugar and modified Ziehl Neelsen staining; and for the factors, documentary analysis of records. Results. Twenty patients had intestinal parasites; 11 with intestinal coccidia, Cystosospora belli (36\%) and Cryptosporidium sp $(14 \%)$. No association was observed between time of diagnosis $(p=0.157)$, CD4 + T lymphocyte count $(p=0.754)$ and viral load $(p=1,000)$, and intestinal coccidiosis and cryptosporidiosis. Conclusions. Intestinal coccidiosis and cryptosporidiosis are frequent infections and cause diarrhea in HIV patients in times of highly active antiretroviral therapy (HAART).

Keywords: Coccidia, Cryptosporidium, Cystoisospora (Source: MeSH-NLM)

\footnotetext{
${ }^{1}$ Hospital Regional Docente Las Mercedes, Lambayeque, Perú.

${ }^{2}$ Biólogo- Microbiólogo, especialista en Microbiología Clínica.
} 


\section{INTRODUCCIÓN}

La diarrea es una complicación común entre los pacientes con el síndrome de la Inmunodeficiencia adquirida (SIDA). La frecuencia varía notablemente entre los diferentes países siendo más frecuente en países en desarrollo, donde se reportan tasas tan altas como del 60 al 90\%. La diarrea crónica es uno de los factores más importantes que contribuyen al desarrollo de malabsorción y desnutrición en el paciente con SIDA y se calcula que su incidencia a lo largo de la vida entre los pacientes infectados con el VIH es de $30-70 \%$ (1).

Dentro de los llamados patógenos intestinales emergentes y reemergentes destaca un grupo de protozoarios parásitos intracelulares conocidos como coccidios intestinales donde se incluyen Cryptosporidium spp., Cystoisospora belli y Cyclospora cayetanensis que causan, respectivamente, criptosporidiosis, cystoisosporosis y ciclosporosis ${ }^{(1)}$.

Es conocida la elevada frecuencia de estas infecciones en pacientes inmunocomprometidos, especialmente en aquellos infectados por el Virus de la Inmunodeficiencia Humana $(\mathrm{VIH})$. En estos pacientes dichas coccidiosis suelen cursar con diarrea y otras manifestaciones que generalmente son graves, incluso fatales (2). En Perú, la diarrea es común en personas con SIDA, y las especies de C. belli y Cryptosporidium spp. se han encontrado en $34 \%$ de las personas en el momento del diagnóstico de SIDA ${ }^{(3)}$.

En el presente estudio tuvo como fin determinar la frecuencia de coccidiosis intestinal y criptosporidiosis en pacientes diarreicos e infección con el virus de la inmunodeficiencia humana, admitidos en un hospital de Chiclayo, Perú; así como, relacionarlo con el tiempo de diagnóstico, linfocitos T CD4+ y carga viral.

\section{MATERIAL Y MÉTODOS}

\section{Tipo y diseño de la investigación}

Se realizó un estudio observacional, prospectivo y transversal analítico, entre los meses de marzo a noviembre del 2015, en el Hospital Regional Docente Las Mercedes (HRDLM) Chiclayo, Perú.

\section{Población y muestra}

La población estuvo conformada por todos los pacientes con el $\mathrm{VIH}$ que presentaron cuadros diarreicos atendidos en el Programa de Control de Infecciones de Transmisión Sexual y Sida (PROCITSS) del HRDLM, la muestra estuvo constituida por 34 pacientes que cumplían con los criterios de inclusión ${ }^{(4)}$.

\section{Técnicas e instrumentos de recolección de datos}

La coccidiosis y criptosporidiosis se estudió en tres muestras de heces seriadas por los métodos de Examen directo, Método de Concentración por flotación Sheather Sugar y el método de Ziehl-Neelsen modificado, siguiendo los proce- dimientos descritos previamente ${ }^{(5)}$. El recuento de linfocitos T CD4+, carga viral y tiempo de diagnóstico se obtuvieron de los registros del PROCITSS del mismo hospital y a través de la página web NETLAB del Instituto Nacional de Salud (INS).

\section{Análisis de datos}

La información recolectada fue organizada en una base de datos en Microsoft Excel 2010 (Microsoft Office $®$ ). Asimismo se realizaron pruebas estadísticas descriptivas para obtener frecuencias absolutas y relativas de las variables. La prueba de Chi cuadrado se utilizó para determinar la asociación de las variables tiempo de diagnóstico, carga viral y recuento de linfocitos T CD4+ con la mayor frecuencia de las parasitosis (coccidiosis intestinal y criptosporidiosis). Se consideró significativo un valor de $p<0,05$ y se usó Microsoft Excel Megastat.

\section{Consideraciones éticas}

El protocolo de estudio fue revisado y recibió la autorización del establecimiento por cuanto no se contaba con un comité de ética. Los pacientes participantes firmaron su consentimiento informado. Se mantuvo la confidencialidad de la información recolectada, la misma que fue de acceso restringido.

\section{RESULTADOS}

En el presente estudio participaron 34 pacientes que cumplían con todos los criterios de selección de los cuales el $85,3 \%$ eran varones y $14,7 \%$ eran mujeres; con un promedio de edad de 33 años (rango de edad 17 a 50 años). La frecuencia general de la parasitosis fue de $64,7 \%$, C. belli 23,5 (8/34) y Cryptosporidium spp. 8,8 \% (3/34) (Tabla 1).

Tabla 1. Frecuencia de coccidiosis intestinal, Cryptosporidium spp. y otros parásitos en pacientes diarreicos e infección por VIH, admitidos en un hospital de Chiclayo, Perú.

\begin{tabular}{lcc} 
Parásitos & $\mathrm{N}$ & $\%$ \\
\hline $\begin{array}{l}\text { Cystoisospora } \\
\text { belli }\end{array}$ & 8 & 23,5 \\
$\begin{array}{l}\text { Cryptosporidium } \\
\text { spp. }\end{array}$ & 3 & 8,8 \\
$\begin{array}{l}\text { Otros protozoos } \\
\text { Helmintos }\end{array}$ & 7 & 20,6 \\
Negativo & 4 & 11,8 \\
Total & 12 & 35,3 \\
\hline
\end{tabular}

En 10 pacientes positivos a coccidiosis intestinal y criptosporidiosis se encontró recuentos de LT CD4 menor a 200 células/uL; asimismo, en cuanto a la carga viral, de los 11 pacientes positivos, cinco tuvieron una carga viral de menos de 200 copias $/ \mathrm{mL}$ (cuatro de ellos parasitados con C. belli y uno con Cryptosporidium spp.) (Tabla 2). 
En cuanto al análisis bivariado, no se encontró asociación entre el tiempo de diagnóstico $(p=0,154)$, el recuento de linfocitos T CD4+ $(p=0,757)$ y carga viral $(p=1,000)$, con la mayor frecuencia de la coccidiosis y criptosporidiosis (tabla 2$)$.

Tabla 2. Asociación entre el tiempo de diagnóstico, linfocitos T CD4+ y carga viral con la coccidiosis intestinal y criptosporidiosis.

\begin{tabular}{llll}
\hline Variable & $\mathrm{N}(\%)$ & Positivo/total $(\%)$ & Valor $\mathrm{p}$ \\
\hline Tiempo de diagnóstico & & & \\
$\leq 2$ años & $20(58,8)$ & $3 / 20(15,0)$ & 0,154 \\
$>$ 2 años & $14(41,2)$ & $8 / 14(57,1)$ & \\
Linfocitos T CD4+ (cel/uL) & & & 0,757 \\
Menor a 200 & $26(76,5)$ & $10 / 26(38,5)$ & \\
Mayor a 200 & $8(23,5)$ & $1 / 8(12,5)$ & \\
Carga viral (copias ARN/ & & & 1,000 \\
mL) & $14(41,2)$ & $5 / 14(35,7)$ & \\
Menor a 200 & $10(29,4)$ & $3 / 10(30,0)$ & \\
200 a 100 mil & $10(29,4)$ & $3 / 10(30,0)$ & \\
100 mil a más & & & \\
\hline
\end{tabular}

\section{DISCUSION}

Los resultados obtenidos en la presente investigación realizada en población $\mathrm{VIH}$ de la región mostraron que la frecuencia de parásitos intestinales fue de $65 \%$, del cual un porcentaje muy significativo correspondió a la infección por coccidiosis y criptosporidiosis, resultados similares obtenidos por otros autores ${ }^{(2,6)}$ en el sentido que la parasitosis causada por coccidios es elevada.

Fueron identificados C. belli (36\%) y Cryptosporidium spp. $(14 \%)$ concordándose con los autores ya mencionados y con otros $(7,8,9,10,11)$; esto debido a que en esta investigación al igual que en las otras los pacientes padecían de diarreas crónicas o prolongadas las que son provocadas principalmente por dichos agentes infecciosos, considerándose incluso a dichos agentes como parásitos emergentes en los pacientes con VIH/SIDA.

El porcentaje de coccidiosis intestinal y criptosporidiosis obtenido es mayor al hallado por otros autores ${ }^{(12,13,14)}$,explicándose esto tanto en la situación de los pacientes en relación con el Tratamiento Antirretroviral de Gran Actividad (TARGA) como en la fase de la infección, así en éste trabajo todos los pacientes en estudio se encontraban con TARGA y el 15 $\%$ de los pacientes positivos para para estas infecciones lo habían abandonado en algún momento del proceso de la enfermedad, siendo posible entonces el incremento de infecciones parasitarias secundarias ${ }^{(16)}$.

Sin embargo en las referencias consideradas los pacientes estaban sujetos al TARGA por tanto de alguna manera las infecciones secundarias estaban controladas ${ }^{\left({ }^{12}\right)}$. Por otro lado en la presente investigación la mayoría de pacientes estaba en la fase SIDA con las mismas consecuencias de la situación anterior, en tanto los pacientes evaluados en Cuba estaban en la etapa de $\mathrm{VIH}$ refrendado esto en la elevada carga viral de los pacientes ${ }^{(13,14)}$. En el recuento de linfocitos TCD $4(+)$ se observó un promedio de 124 células/ml y en más del $90 \%$ de pacientes un conteo menor a 200 células $/ \mathrm{ml}$, resultados similares reportados por ${ }^{(3,6,6,8,13)}$.

Por lo expresado se deduce que las características de los pacientes inmunodeprimidos con un recuento de linfocitos $T$ CD4(+) menor a 200 células/uL siguen un patrón regular, aun así en relación a la parasitosis, ésta, si bien se presenta asociada a la infección, la prevalencia y la presencia de algunos tipos de parásitos pueden estar influenciadas por factores epidemiológicos, destacándose que $C$. belli y Cryptosporidium spp. siempre están presentes. Al respecto, un estudio previo realizado en una serie de casos clínicos de cistoisoporosis en pacientes con infección VIH y diarrea recurrente, reportó distinta evolución clínica, donde algunos pacientes podrían experimentar infección persistente y diarrea recurrente a pesar de una adecuada respuesta al TARGA y profilaxis secundaria con cotrimoxazol ${ }^{(16)}$.

De los datos de la ficha de información se obtiene que todos los pacientes del estudio tenían los servicios básicos de agua y desagüe por tanto consumían agua potable; aun así debe considerarse que Cryptosporidium spp. posee una alta capacidad de sobrevivencia en el ambiente y en el agua, incluyendo el agua potabilizada con cloro (al igual que Giardia lamblia), lo que confirma su presencia en éste estudio.

A pesar que algunos estudios sugieren que la parasitosis en este tipo de pacientes puede estar relacionada con el consumo de agua no potable y de alimentos crudos así como con la ten 
encia de animales y los malos hábitos higiénicos sanitarios (15); deben considerarse otros factores como el oficio del paciente y las diferentes prácticas sexuales (felación en pacientes homosexuales).

En conclusión la frecuencia de coccidiosis intestinal y criptosporidiosis en pacientes diarreicos e infección con el virus de la inmunodeficiencia humana del HRDLM fue de $32 \%$. Así mismo, no se encontró asociación entre la coccidiosis intestinal y criptosporidiosis identificados y las variables estudiadas (tiempo de diagnóstico, recuento de linfocitos $T$ CD4+ y carga viral).

\section{REFERENCIAS BIBLIOGRAFICAS}

1. García C, Rodríguez E, Do N, López D, Terashima A, Gotuzzo E. Parásitos intestinales en pacientes con infección VIH - SIDA. Perú. Rev Gastroenterol. 2006; 26(1): 21-4.

2. Requena I, Añez A, Lacourt E, Blanco $Y$, Castillo H, Rivera M, Devera R. Elevada prevalencia de coccidios intestinales en pacientes infectados con el Virus de la Inmunodeficiencia Humana en Ciudad Bolívar, Venezuela. Rev Biomed. 2007; 18(1):73-5

3. Carcamo C, Hooton T, Wener M, Weiss N, Gillman R, Arévalo J. Etiologies and manifestations of persistent diarrhea in adults with HIV-1 infections: A case-control study in Lima, Peru. The Journal of infectious Diseases. 2005; 191(1): 11-9.

4. Alvitres V. Método Científico. Planificación de la Investigación. Chiclayo. Perú. 2000. 2da Edición. Editorial Ciencia.

5. Beltran M, Tello R, Naquira C. Manual de Procedimientos de laboratorio para diagnóstico de los parásitos intestinales del hombre. Instituto $\mathrm{Na}$ cional de Salud del Perú. 2003:89p.

6. Hurtado J, Lagunes V, Ortigoza S, Cortes C, Torres B, Rodríguez E. Cyclospora cayetanensis y Cryptosporidium sp., principales parásitos en pacientes con SIDA. Perú. Rev Redalyc org. 2009; 34(1):106.

7. Cinerman S, Cinerman B, Salomao D. Enteric parasites and AIDS. Sao Paulo. Brasil. Rev Paul Med 1999; 117(6): 266-73

8. Sorto R y Bu E. Perfil clínico parasitológico de pacientes con VIH/SIDA y diarrea crónica atendidos en el hospital escuela. Rev Med Hondur. 2006; 74(2): 69-76.

9. Rivero Z, Hernández A, Bracho A, Salazar S, Villalobos R. Prevalencia de microsporidios intestinales y otros enteroparásitos en pacientes con VIH positivo de Maracaibo, Venezuela. Biomédica. 2013; 33 (1):638-45.

10.Guerra da Rocha L y Andrade do Santos T. Isospora belli en los pacien tes con SIDA - Brasil. Parasitol. Latinoam. 2002; 57 (3 - 4):161-5.

11.Prasad K, Nag V, Dhole T, Ayyagari A. Identificación de patógenos entéricos en pacientes con VIH positivo con diarrea en el Norte de la India. 2000; 18(1): 23-6.

12. Montalvo R, Ticona E, Ñavincopa M, Garcia Y, Chavez V, Arevalo J, Soria J, Huiza A. Diarrea recurrente por Cystoisospora belli en pacientes con infección por VIH con TARGA. Rev Med Exp Salud Pública. 2013; 30(2):326-30.

13.Aillen G, Palma S, Molina R, Borrero $Y$, Fernández $Y$, Pinillos T, Velásquez $\mathrm{B}$. Microorganismos más frecuentes en pacientes con virus de Inmunodeficiencia Humana tipo 1 ( $\mathrm{VIH}-1)$ positivos cubanos con elevados niveles de carga Viral. Medigraphic. 2006; 31(1): 85-99.

14.Capó de Paz. V, M. Barreto, V. Beltrán, C. Luzardo, A. Martínez, Z. Alujas. Diagnóstico de coccidias y microsporas en muestras de heces diarreicas de pacientes cubanos seropositivos al VIH: primer reporte de microsporas en cuba. Rev Cubana Med Trop. 2003; 55(1):8-14.

15.Vivas M, Castro C, Delgado M. Búsqueda de Isospora belli en heces de pacientes con síndrome de Inmunodeficiencia adquirida y que presentan diarrea - Yucatán. México. Rev. Fac. Med UNAM 2009; 52(5):2047 .

16.Silva-Díaz H, Fernández-Valverde D, Hernández-Córdova G, Failoc-Rojas VE. Infección por Cystoisospora belli en pacientes con VIH: análisis de casos con diferente evolución clínica. Rev Chil infectología. 2017; 34(4):347-51. 